\title{
Assessing the Similarity of Quantum Images based on Probability Measurements
}

\author{
Fei Yan, Phuc Q. Le, Abdullah M. Iliyasu, Bo Sun, Jesus A. Garcia, Fangyan Dong, Kaoru Hirota \\ Department of Computational Intelligence and Systems Science \\ Tokyo Institute of Technology \\ Yokohama, JAPAN \\ yan@hrt.dis.titech.ac.jp
}

\begin{abstract}
A method to analyze the similarity between two quantum images of the same size is proposed based on a representation for the quantum images. The similarity value is estimated according to the probability distribution of the results from quantum measurements. The proposed method is fast because a single operation can transform the entire information encoded in two images simultaneously. Two simulation-based experiments, which provide a reasonable estimation to the quantum images' similarity, are implemented using Matlab on a classical computer by means of linear algebra with complex vectors as quantum states and unitary matrices as unitary transformations. It also opens the door towards image searching from a database on quantum computers.
\end{abstract}

Keywords-quantum computation; image processing; quantum image; image similarity; probability measurement

\section{INTRODUCTION}

Quantum computation is a promising and rapidly growing field [1][2][4]. During the last decade, a series of significant breakthroughs have been made. In 1994 Peter Shor discovered a quantum algorithm to factor integer numbers in polynomial time [1]. Two years later, Gover proved that a quantum computer could search an unsorted database in the square root of the classical time [2]. These results provide solid evidence of the strength of quantum computers over classical ones. In quantum computation, quantum circuit models are very important because any unitary operation or quantum algorithm can be decomposed into a circuit consisting by a succession of basic unitary gates that act on one or two qubits only [3]. Many elementary gates including single qubit gates, controlled-NOT gate, and Toffoli gate for quantum computation are introduced in [4].

Research on quantum image processing started with proposals on quantum image representations such as Qubit Lattice [5], Real Ket [6], and the Flexible Representation of Quantum Image (FRQI) [7]. Some operations can be done through applying the elementary gates such as Pauli-X and Hadamard gates combined with appropriate quantum measurement [8] on these representations. For example, several processing transformations were proposed based on the FRQI representation such as the geometric transformations, GTQI [3] and the CTQI [9], which focuses on the color information. Moreover, some applications, such as a scheme to watermark and authenticate quantum images [10] and a framework to produce movies on quantum computers [11], have been studied based on this representation.

Most of the operations [3][9][10], however, are focused on single images. This paper presents a modest attempt to compare two quantum images encoded in the FRQI representation. Succinctly put, the main contributions of this work is confirming the relationship between the similarity value of two quantum images and the probability of a state on an additional wire in the quantum circuit.

The comparison process is fast and costless since only a single quantum gate without any control-condition could transform the entire information encoding the two quantum images simultaneously. In addition, the two quantum images being compared remain intact prior to the quantum measurement. Since in practice, the state of the quantum system should be determined by the quantum measurement. The measurement, however, would destroy the superposition state in the system, so it is necessary to repeat the construction of the state $n(n>1)$ times, and measure each one to summarize the measurement results from which we can read the similarity value between the two images.

The rest of the paper is organized as follows. Representation of strip to encode two or more quantum images is described in section 2. Similarity between two quantum images is analysed in section 3. Simulation experiments based on the proposed method are reported in section 4 . Finally, the remarks and future perspectives are discussed in section 5 .

\section{REPRESENTATION OF STRIP COMPRISING QUANTUM IMAGES}

The basic notations used in this paper are introduced below. The state of a quantum system is described as a vector in a Hilbert space which is called a ket in quantum mechanical notation. The ket and its adjoint, bra, notations are defined as

$$
\begin{gathered}
|u\rangle=\left[\begin{array}{c}
u_{0} \\
u_{1} \\
\vdots \\
u_{n-1}
\end{array}\right], u_{i} \in C, i=0,1, \cdots n-1, \\
\langle u|=| u\rangle^{*}=\left[\begin{array}{llll}
u_{0}^{*} & u_{1}^{*} & \cdots & u_{n-1}^{*}
\end{array}\right],
\end{gathered}
$$

where $u^{*}$ is the notation of complex conjugate of $u$. 
The smallest unit of information in a quantum system is a qubit, which is a unit vector in two dimensional Hilbert space defined by

$$
\left[\begin{array}{l}
\alpha \\
\beta
\end{array}\right]=\alpha\left[\begin{array}{l}
1 \\
0
\end{array}\right]+\beta\left[\begin{array}{l}
0 \\
1
\end{array}\right]=\alpha|0\rangle+\beta|1\rangle,
$$

where $\alpha$ and $\beta$ are complex numbers.

The notation for the tensor product, $\otimes$, is used to express the composition of quantum systems. The tensor product of two matrices is defined as follows:

$$
\begin{gathered}
A=\left[\begin{array}{cccc}
a_{11} & a_{12} & \cdots & a_{1 m} \\
a_{21} & a_{22} & \cdots & a_{2 m} \\
\vdots & \vdots & \ddots & \vdots \\
a_{n 1} & a_{n 2} & \cdots & a_{n m}
\end{array}\right], B=\left[\begin{array}{cccc}
b_{11} & b_{12} & \cdots & b_{1 q} \\
b_{21} & b_{22} & \cdots & b_{2 q} \\
\vdots & \vdots & \ddots & \vdots \\
b_{p 1} & b_{p 2} & \cdots & b_{p q}
\end{array}\right], \\
A \otimes B=\left[\begin{array}{cccc}
a_{11} B & a_{12} B & \cdots & a_{1 m} B \\
a_{21} B & a_{22} B & \cdots & a_{2 m} B \\
\vdots & \vdots & \ddots & \vdots \\
a_{n 1} B & a_{n 2} B & \cdots & a_{n m} B
\end{array}\right] .
\end{gathered}
$$

The short notation for tensor product $|u\rangle \otimes|v\rangle$ of two vectors or two kets, $|u\rangle$ and $|v\rangle$, is $|u v\rangle$ or $|u\rangle|v\rangle$ and $A^{\otimes}=A \otimes A \otimes \cdots \otimes A$ is used to denote the tensor product of a matrix A for $n$ times.

In the quantum circuit model, a complex transform is broken down into simpler gates such as the commonly used NOT, Hadamard, CNOT, and Toffoli gates which are shown in Fig. 1. The gate which acts on $k$ qubits is usually represented by a $2^{k} \times 2^{k}$ unitary matrix, and the number of qubits in the input and output of the gate have to be equal.

The final step in quantum simulation is the measurement which converts the quantum information into the classical information in form of probability distributions, i.e., it converts a single qubit state $|\psi\rangle=\alpha|0\rangle+\beta|1\rangle$ into a probabilistic classical bit $M$ (distinguished from a qubit by drawing it as a double-line wire), which is 0 with probability $|\alpha|^{2}$, or 1 with probability $|\beta|^{2}$ as shown in Fig. 2.

$$
\begin{gathered}
\text { NOT Gate } \\
\alpha|0\rangle+\beta|1\rangle-X-\beta|0\rangle+\alpha|1\rangle \\
\text { Hadamard Gate } \\
\alpha|0\rangle+\beta|1\rangle-H-\alpha \frac{(|0\rangle+|1\rangle)}{\sqrt{2}}+\beta \frac{(|0\rangle-|1\rangle)}{\sqrt{2}}
\end{gathered}
$$

\section{Controlled NOT or CNOT Gate}
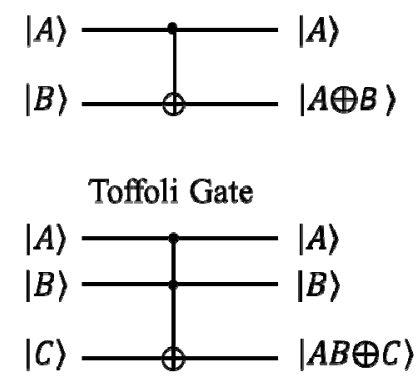

Figure 1. NOT, Hadamard, CNOT, and Toffoli gates

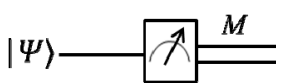

Figure 2. Quantum circuit symbol for measurement

The flexible representation for quantum images, FRQI, captures the essential information about the colors as well as the corresponding positions of every point in an image and integrates them into a quantum state having its formula in (1),

$$
\begin{gathered}
|I(\theta)\rangle=1 / 2^{n} \sum_{i=0}^{2^{2 n}-1}\left|c_{i}\right\rangle \otimes|i\rangle, \\
\left|c_{i}\right\rangle=\cos \theta_{i}|0\rangle+\sin \theta_{i}|1\rangle, \\
\theta_{i} \in[0, \pi / 2], \quad i=0,1, \cdots 2^{2 n}-1,
\end{gathered}
$$

where $|0\rangle$ and $|1\rangle$ are 2-D computational basis quantum states, $|i\rangle, i=0,1, \cdots 2^{2 n}-1$ are $2 \mathrm{n}-\mathrm{D}$ computational basis quantum states and $\theta=\left(\theta_{0}, \theta_{1}, \cdots \theta_{2^{2 n-1}}\right)$ is the vector of angles encoding colors. There are two parts in the FRQI representation of an image; $\left|C_{i}\right\rangle$ and $|i\rangle$ which encode information about the colors and their corresponding positions in the image, respectively. An example of a $2 \times 2$ image is shown in Fig. 3. The FRQI state is a normalized state, i.e. $\| I(\theta)\rangle \|=1$ as given by

$$
\| I(\theta)\rangle \|=1 / 2^{n} \sqrt{\sum_{i=0}^{2^{2 n}-1}\left(\cos ^{2} \theta_{i}+\sin ^{2} \theta_{i}\right)}=1 .
$$

In order to compare two $n \times n$ quantum images, a natural instinct would be to compare the colors in the two quantum images position-by-position as shown in Fig. 4. However, it is impossible to conclusively identify two unknown pure quantum states as being identical [12], which means comparing the color information directly in two quantum images is not allowed. Therefore, a new representation to compare quantum images of the same size is introduced as shown in Definition 1.

Definition 1 A strip [11], $|S(m, n)\rangle$, is an array comprising $2^{m}$ FRQI sub-images. The state of this strip can be defined by

$$
\begin{gathered}
|S(m, n)\rangle=1 / 2^{m / 2} \sum_{j=0}^{2^{m}-1}\left|I_{j}\right\rangle \otimes|j\rangle, \\
\left|I_{j}\right\rangle=1 / 2^{n} \sum_{i=0}^{2^{2 n}-1}\left|c_{j, i}\right\rangle \otimes|i\rangle,
\end{gathered}
$$

where $\left|I_{j}\right\rangle$ is a FRQI image as defined in (1), $m$ is the number of qubits required to encode the images being compared, $|j\rangle$ is the position of each image in the strip. A strip can be horizontally-oriented or vertically-oriented. The latter case, vertically-oriented strip, is implied throughout the discussion. $|S(m, n)\rangle$ is also in a normalized state which can be shown by

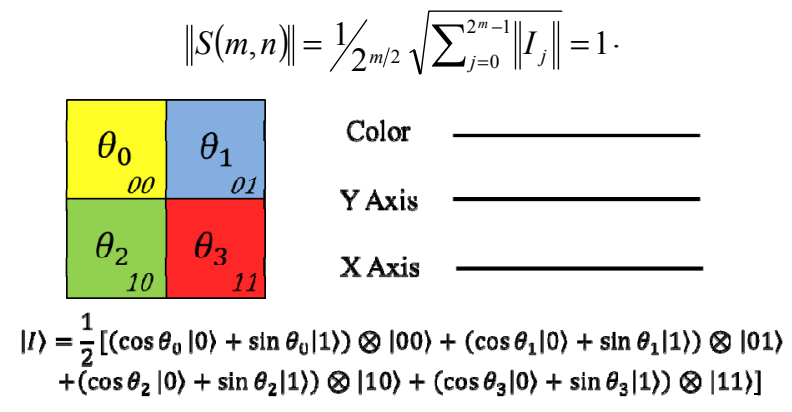

\footnotetext{
Figure 3 . A $2 \times 2$ image, its circuit structure and FRQI state
} 


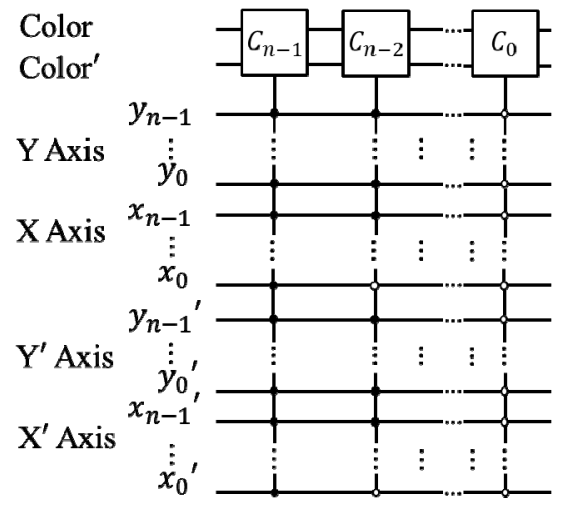

Figure 4. Impossible way to compare two quantum images

To encode only two images being compared, we set $m=1$. Thus, (5) can be rewritten by

$$
|S(1, n)\rangle=1 / \sqrt{2}\left(\left|I_{0}\right\rangle \otimes|0\rangle+\left|I_{1}\right\rangle \otimes|1\rangle\right),
$$

where

$$
\left|I_{0}\right\rangle=1 / 2^{n} \sum_{i=0}^{2^{2 n-1}}\left(\cos \theta_{i}|0\rangle+\sin \theta_{i}|1\rangle\right) \otimes|i\rangle
$$

and

$$
\left|I_{1}\right\rangle=1 / 2^{n} \sum_{i=0}^{2^{2 n}-1}\left(\cos \varphi_{i}|0\rangle+\sin \varphi_{i}|1\rangle\right) \otimes|i\rangle
$$

are the two quantum images being compared, which are located in the upper part and lower part of the strip respectively for the vertically-oriented strip encoding only two images.

An example that two $2 \times 2$ images $\left|I_{0}\right\rangle$ and $\left|I_{1}\right\rangle$ are combined vertically to a strip is shown in Fig. 5 . Together with the combined image, a strip wire is applied on the circuit of this quantum system to represent upper and lower image.

\section{COMParison OF Similarity BetweEn Two Quantum IMAGES}

Some operations are done to the quantum system constituted by two $n \times n$ quantum images so as to get the similarity value between them. A Hadamard gate $H=1 / \sqrt{2}\left(\begin{array}{cc}1 & 1 \\ 1 & -1\end{array}\right)$, which maps the basis state $|0\rangle$ to $(|0\rangle+|1\rangle) / \sqrt{2}$ and $|1\rangle$ to $(|0\rangle-|1\rangle) / \sqrt{2}$ is applied on the strip wire to obtain the recombination of $\left|I_{0}\right\rangle$ and $\left|I_{1}\right\rangle$, then it is followed by a measurement operation as shown in Fig. 6.

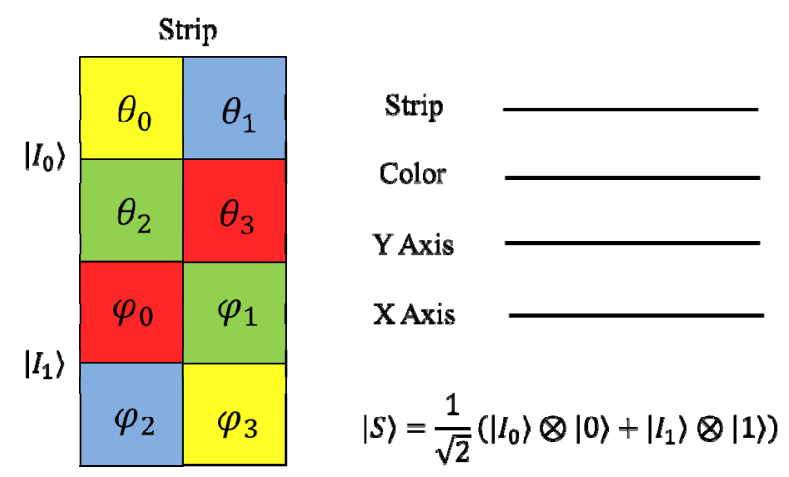

Figure 5. A strip encoding two $2 \times 2$ images, its circuit structure and FRQI state

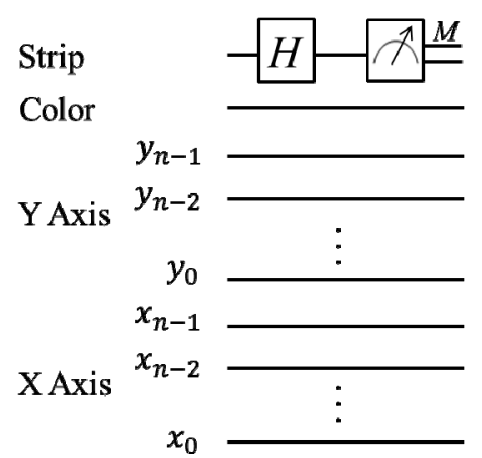

Figure 6. Generalized circuit for comparing two quantum images

Corresponding to the circuit shown in Fig. 6, the state of quantum system after applied the Hadamard gate on the strip wire can be shown as follows according to (8), (9) and (10):

$$
\begin{aligned}
& H|S(1, n)\rangle=1 / \sqrt{2}\left(\left|I_{0}\right\rangle \otimes H|0\rangle+\left|I_{1}\right\rangle \otimes H|1\rangle\right) \\
& \left.=1 / 2\left[I_{0}\right\rangle \otimes(|0\rangle+|1\rangle)+\left|I_{1}\right\rangle \otimes(|0\rangle-|1\rangle)\right] \\
& =1 / 2\left[\left(\left|I_{0}\right\rangle+\left|I_{1}\right\rangle\right) \otimes|0\rangle+\left(\left|I_{0}\right\rangle-\left|I_{1}\right\rangle\right) \otimes|1\rangle\right] \\
& \left.=1 / 2^{n+1}\left\{\sum_{i=0}^{2^{2 n-1}}\left[\left(\cos \theta_{i}+\cos \varphi_{i}\right)|0\rangle+\left(\sin \theta_{i}+\sin \varphi_{i}\right)|1\rangle\right] i\right\rangle\right\} \otimes|0\rangle \\
& \left.+1 / 2^{n+1}\left\{\sum_{i=0}^{2^{2 n-1}}\left[\left(\cos \theta_{i}-\cos \varphi_{i}\right)|0\rangle+\left(\sin \theta_{i}-\sin \varphi_{i}\right)|1\rangle\right] i\right\rangle\right\} \otimes|1\rangle .
\end{aligned}
$$

Obviously, the result of the measurement depends on the disparities between $\left|I_{0}\right\rangle$ and $\left|I_{1}\right\rangle$. The probability of state $|0\rangle$ on the strip wire is shown by

$$
\begin{aligned}
\operatorname{Pr}(|0\rangle) & =\left(1 / 2^{n+1}\right)^{2} \sum_{i=0}^{2^{2 n-1}}\left[\left(\cos \theta_{i}+\cos \varphi_{i}\right)^{2}+\left(\sin \theta_{i}+\sin \varphi_{i}\right)^{2}\right] \\
& =1 / 2^{2 n+1} \sum_{i=0}^{2^{2 n-1}}\left[1+\cos \left(\theta_{i}-\varphi_{i}\right)\right] .
\end{aligned}
$$

In the same way, that of state $|1\rangle$ is given by

$$
\operatorname{Pr}(|1\rangle)=1 / 2^{2 n+1} \sum_{i=0}^{2^{2 n}-1}\left[1-\cos \left(\theta_{i}-\varphi_{i}\right)\right] .
$$

Notice that the probabilities sum up to $1, \operatorname{Pr}(|0\rangle)+\operatorname{Pr}(|1\rangle)=1$, as they should.

Definition 2 Pixel difference in position $i, \sigma_{i}$, is defined by

$$
\sigma_{i}=\left|\theta_{i}-\varphi_{i}\right|
$$

where $\theta_{i}$ and $\varphi_{i}$ represent the color information in $\left|I_{0}\right\rangle$ and $\left|I_{1}\right\rangle$ respectively, $\sigma_{i} \in[0, \pi / 2]$.

It is obvious that the pixel difference $\sigma_{i}$ is related to the probability of getting readout of $1, \operatorname{Pr}(1\rangle)$, in the measurement and $\operatorname{Pr}(1\rangle)$ will increase when pixel difference increase. Furthermore, the similarity between two images, which is the sum of pixel difference in every position, depends on $\operatorname{Pr}(|1\rangle)$.

Definition 3 Similarity between two quantum images $\left|I_{0}\right\rangle$ and $\left|I_{1}\right\rangle$ as defined in (9) and (10) is

$$
\operatorname{sim}\left(\left|I_{0}\right\rangle,\left|I_{1}\right\rangle\right)=1-2 \operatorname{Pr}(|1\rangle),
$$

where $\operatorname{Pr}(|1\rangle)$ is defined in (13), $\operatorname{sim}\left(\left|I_{0}\right\rangle,\left|I_{1}\right\rangle\right) \in[0,1]$. 


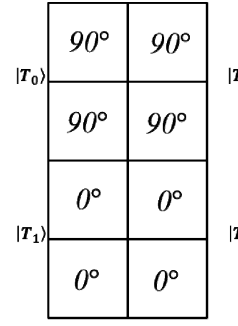

$\operatorname{Pr}_{1}(|1\rangle)=0.50$

Figure 7. Analysis of comparing quantum images through similarity value

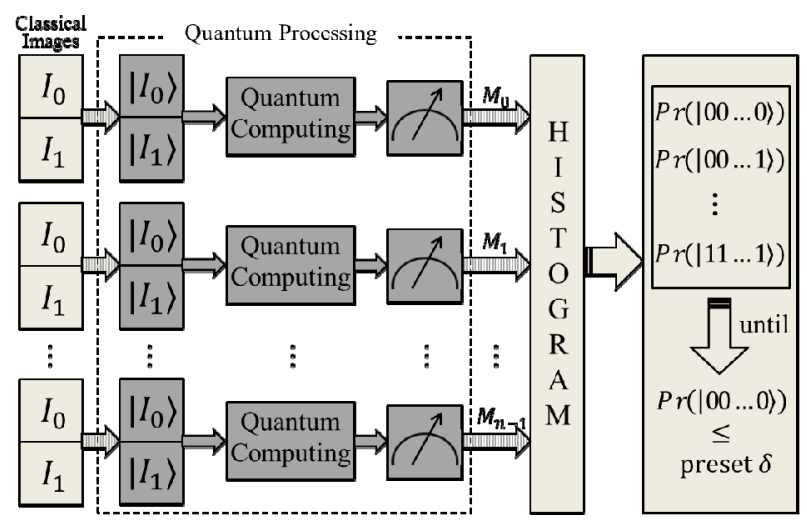

Figure 8. Block diagram of procedure to compare two images on quantum computers

Some special cases about the similarity between two quantum images are listed as follows:

a) if $\forall \sigma_{i}=0$, then $\operatorname{sim}\left(\left|I_{0}\right\rangle,\left|I_{1}\right\rangle\right)=1$;

b) elseif $\forall \sigma_{i}=\pi / 2$, then $\operatorname{sim}\left(\left|I_{0}\right\rangle,\left|I_{1}\right\rangle\right)=0$;

c) else $0<\operatorname{sim}\left(\left|I_{0}\right\rangle,\left|I_{1}\right\rangle\right)<1$.

where $i=0,1, \cdots 2^{2 n}-1, \sigma_{i}$ is the pixel difference in position $i$ defined in Definition 2.

An example that comparing image $\left|T_{0}\right\rangle$ with $\left|T_{1}\right\rangle,\left|T_{2}\right\rangle,\left|T_{3}\right\rangle$ and $\left|T_{4}\right\rangle$ is given in Fig. 7. It is easily found that $\operatorname{sim}\left(\left|T_{0}\right\rangle,\left|T_{1}\right\rangle\right)<\operatorname{sim}\left(\left|T_{0}\right\rangle,\left|T_{2}\right\rangle\right)<\operatorname{sim}\left(\left|T_{0}\right\rangle,\left|T_{3}\right\rangle\right)<\operatorname{sim}\left(\left|T_{0}\right\rangle,\left|T_{4}\right\rangle\right)$, actually, $\left|T_{0}\right\rangle=\left|T_{4}\right\rangle$.

However, the quantum state cannot be practically observed in quantum system, so the probability of getting readout of 0 and 1 on the strip wire should be determined through the quantum measurement. But even so, this work is impossible to be finished once since a measurement would destroy the superposition. What can be done is repeating the construction of the state $n(n>1)$ times, and measure each one in order to summarize some sort of histogram as shown in Fig. 8.

The measurement results on the strip wire follow a binomial distribution when $n$ experiments are done. The probability of getting $k$ readouts of 1 in $n$ experiments is given by the probability mass function

$$
\operatorname{Pr}(X=k)=C_{n}^{k} p^{k}(1-p)^{n-k},
$$

where $X$ is the incident that the result of measurement is 1 , $p=\operatorname{Pr}(|1\rangle)$ is expressed in $(13), k=0,1, \ldots n$. The measurement will be continued until $\operatorname{Pr}(X=k) \leq \delta, \delta \in(0,1)$ is a pre-set threshold, which can be read as the reasonable estimation for $\operatorname{Pr}(|00 \cdots 0\rangle)$ in the measurements.

\section{EXPERIMENTS TO ASSESS SIMILARITY BETWEEN TWO IMAGES}

A desktop computer with Intel Core i7, 2 Duo $2.80 \mathrm{GHz}$ CPU, 4GB RAM and 64bit operating system is used to simulate the experiment on quantum images. The simulation is based on linear algebra with complex vectors as quantum states and unitary matrices as unitary transforms using Matlab, and the program is realized by means of (8)-(15) as well as the Definition 2 and 3 which are introduced above. The final step is that of measurement which converts the quantum information to the classical form as probability distributions. Extracting and analyzing the distributions gives information for comparing two quantum images.

The first experiment is that to compare two $8 \times 8$ synthetic images and two $256 \times 256$ synthetic images having 4 gray levels, comprising of black, dark, light, and white colors respectively, as shown in Fig. 9 and Fig. 10. The purpose of this experiment is to analyze the relation between the similarity value of two images and the size of them.

The comparison between the two $8 \times 8$ images is finished based on the circuit in Fig. 11. The circuit includes 8 qubits of which 6 are used to address positions in the image, 1 is applied to store colors, and the remaining qubit is prepared for representing the strip wire where the Hadamard gate and measurement are applied. The similarity value is 0.31 after using the proposed comparing method. On the other hand, the similarity value between the two $256 \times 256$ images is 0.25 , whose circuit comprising of 18 wires is shown in Fig. 13. The two sets of data are given in Table I. It is concluded from the experiment that the similarity between two quantum images will increase along with the size of them.
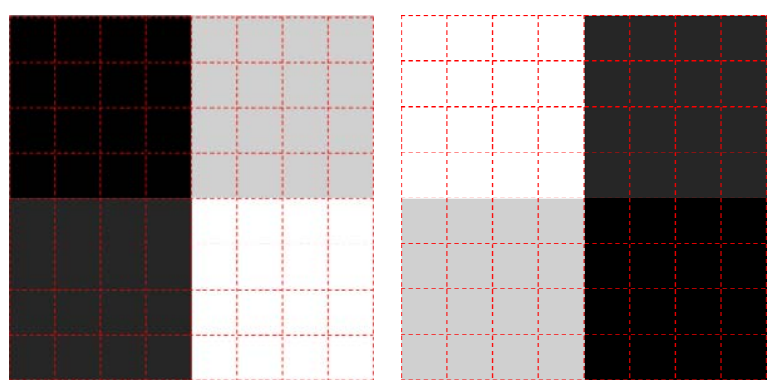

Figure 9 . Two synthetic $8 \times 8$ images

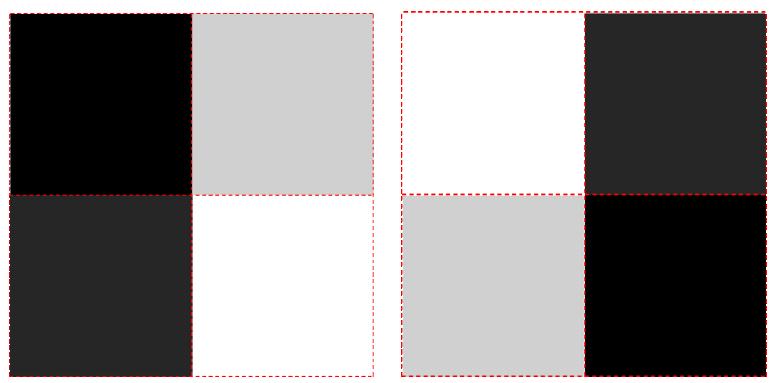

Figure 10. Two synthetic $256 \times 256$ images 


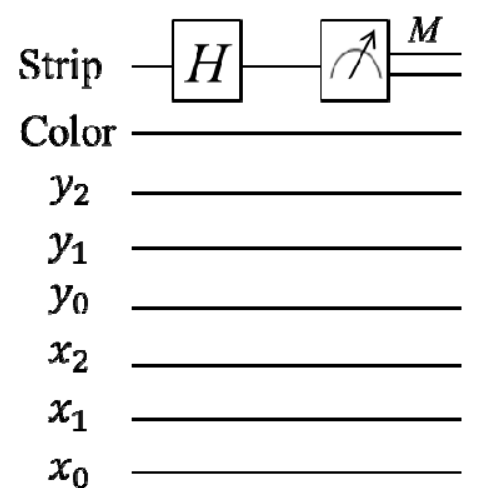

Figure 11. Circuit for comparing two $8 \times 8$ quantum images

TABLE I. RESUlts of COMPARING SyNTHETIC IMAGES

\begin{tabular}{|c|c|c|c|}
\hline Size & No. of qubits (wires) & No. of gates & Similarity value \\
\hline $8 \times 8$ & 8 & 1 & 0.31 \\
\hline $256 \times 256$ & 18 & 1 & 0.25 \\
\hline
\end{tabular}

In the second experiment, a $256 \times 256$ gray scale version of the Lena image labeled $(a)$ in Fig. 12 is considered as the original image. Our aim is to compare it with the other three images, which are $(b)$ its watermarked version, $(c)$ its darkened version and $(d)$ a version that is exactly the same with the original.

The quantum circuit is shown in Fig. 13, which is the same with that of the comparison between two $256 \times 256$ synthetic images. The probabilities of getting the readout of 1 on the strip wire, as well as the similarities among different pairs of images being compared are shown in the Table II. It is obvious that $\operatorname{sim}(a, c)<\operatorname{sim}(a, b)<\operatorname{sim}(a, d)$, actually, image $(a)$ is identical with image $(b)$. The similarity value between $(a)$ and $(c)$ is less than that between $(a)$ and $(b)$ for the important reason that all the pixels are considered in the proposed method. As shown in Fig. 13 the operation to compare two images is realized by using only a single gate. Such an operation, however, can only be achieved on a classical computer by comparing the color of every position one at a time.

The foregoing experiments provide the foundation for the next step in quantum image processing based on the FRQI representation. The results as indicated in this section show that the comparison of two quantum images is feasible and practical. Furthermore, the target area to apply the proposed method is quantum image searching from a database.

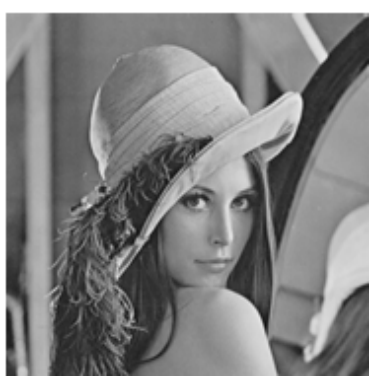

(a) original image

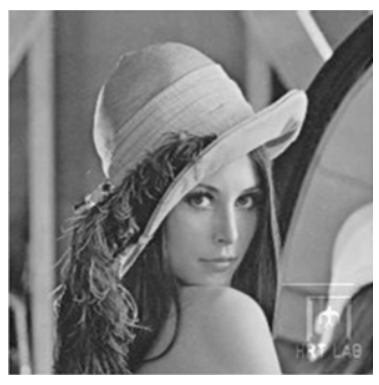

(b) watermarked version

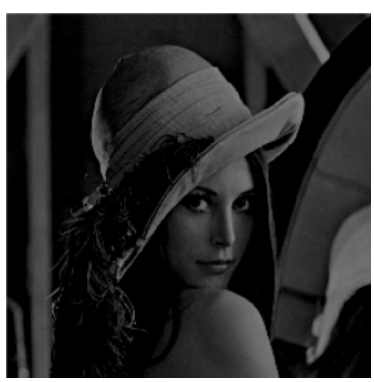

(c) darkened version

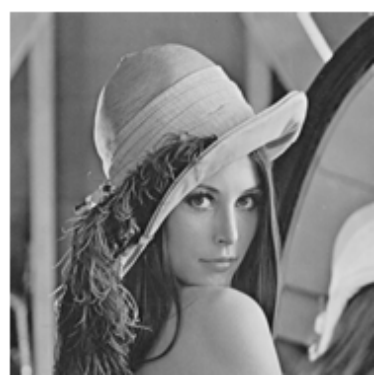

(d) the same with the original
Figure 12. The original Lena image and the other compared images

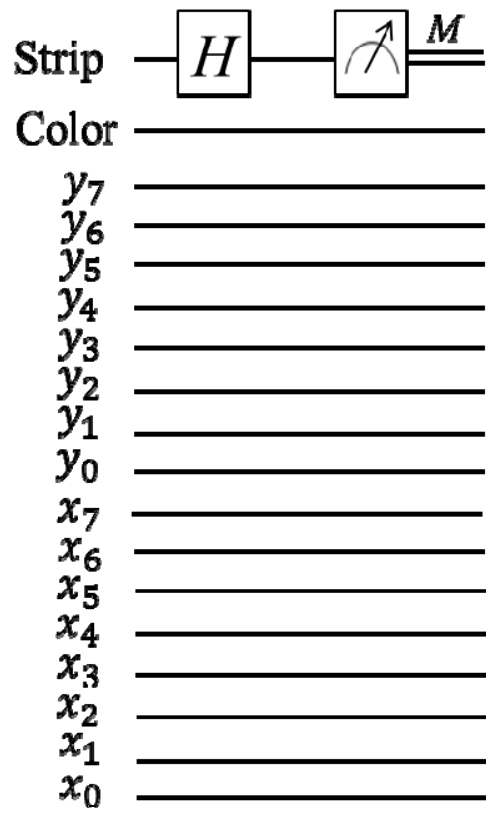

Figure 13. Circuit for comparing two $256 \times 256$ quantum images

TABLE II. RESULTS OF COMPARING THE LENA IMAGES

\begin{tabular}{|c|c|c|c|}
\hline $\begin{array}{c}\text { Images comparison } \\
256 \times 256\end{array}$ & No. of qubits (wires) & $\operatorname{Pr}(11)$ & Similarity value \\
\hline (a), (b) & 18 & 0.005 & 0.990 \\
\hline (a), (c) & 18 & 0.075 & 0.850 \\
\hline (a), (d) & 18 & 0 & 1 \\
\hline
\end{tabular}

\section{CONCLUSIONS}

A method to analyze the similarity between two FRQI quantum images of the same size is proposed. According to the representation of the strip which is combined by two quantum images being compared and the basic operations in the quantum computation, the similarity value is estimated on the basis of the probability distribution of the results from quantum measurements. The proposed method is fast and costless since single quantum gate without any control operation could transform the entire information encoded in two quantum images simultaneously.

Two simulation-based experiments are implemented using Matlab on a classical computer by means of linear algebra with complex vectors as quantum states and unitary matrices as unitary transformations provide a reasonable estimation to the 
image comparison. It is concluded that the similarity between two quantum images depends on the entire information in the both images instead of some parts of them, and furthermore, it is related to the size of the two images.

Since in practice, the state of a quantum system cannot be read directly because a measurement would destroy the superposition. And what's worse, it is not allowed to make copies of the state and measure each one due to the NonCloning theorem. Hence, the construction of the state have to be repeated $n(n>1)$ times, and measure each one to summarize some sort of histogram from which the similarity can be read between two quantum images.

As for the future work, the strip wire on the quantum circuit should be extended so that the proposed comparing method could be applied to more quantum images. And also, we can prepare more original images to achieve the quantum image searching from a database.

\section{REFERENCES}

[1] P. W. Shor, "Algorithms for quantum computation: discrete logarithms and factoring," Proc. 35th Ann. Symp. Found. Comput. Sci. IEEE Computer Soc. Press, Los Almitos, CA. pp. 124-134, 1994.

[2] L. Grover, "A fast quantum mechanical algorithm for database search," Proc. 28th Ann. ACM Symp. Theory Comput. (STOC 1996), ACM, New York, pp. 212-219, 1996.
[3] P. Q. Le, A. M. Iliyasu, F. Dong, and K. Hirota, "Fast geometric transformations on quantum images," IAENG Intl. J. of Applied Mathematics, Vol. 40, No. 3, pp. 113-123, 2010.

[4] A. Barenco, C. H. Bennett, R. Cleve, D. P. DiVincenzo, N. Margolus, P. Shor et al, "Elementary gates for quantum computation," Phys. Rev. A 52, pp. 3457-3467, 1995.

[5] S.E. Venegas-Andraca and S. Bose, "Storing, processing and retrieving an image using quantum mechanics," Proc. SPIE Conf. Quantum Info. and Comput., Vol. 5105, pp. 134-147, 2003.

[6] J. I. Latorre, "Image compression and entanglement," arXiv: quant$\mathrm{ph} / 0510031,2005$.

[7] P. Q. Le, F. Dong, and K. Hirota, "A flexible representation of quantum images for polynomial preparation, image compression, and processing operations," Quantum Info. Proc., Vol. 10, No. 1, pp. 63-84, 2010.

[8] A. M. Childs, D. W. Leung, and M. A. Nielsen, "Unified derivations of measurement-based schemes for quantum computation," Phys. Rev. A 71, 032318, pp. 1-14, 2005.

[9] P. Q. Le, A. M. Iliyasu, F. Dong, and K. Hirota, "Efficient color transformations on quantum image," J. of Adv. Comp. Intl. Info. (JaCIII), Vol. 15, No. 6, pp. 698-706, 2011.

[10] A. M. Iliyasu, P. Q. Le, F. Dong, and K. Hirota, "Watermarking and authentication of quantum images based on restricted geometric transformatinos," Info. Sci., Vol. 186, No. 1, pp. 126-149, 2011.

[11] A. M. Iliyasu, P. Q. Le, F. Dong, and K. Hirota, "A framework for representing and producing movies on quantum computers," Intl. J. of Quantum Info., Vol. 9, No. 6, pp. 1459-1497, 2011.

[12] S. M. Barnett, A. Chefles, and I. Jex, "Comparison of two unknown pure quantum states,” Phys. Letters A, Vol. 307, No. 4, pp. 189-195, 2003. 